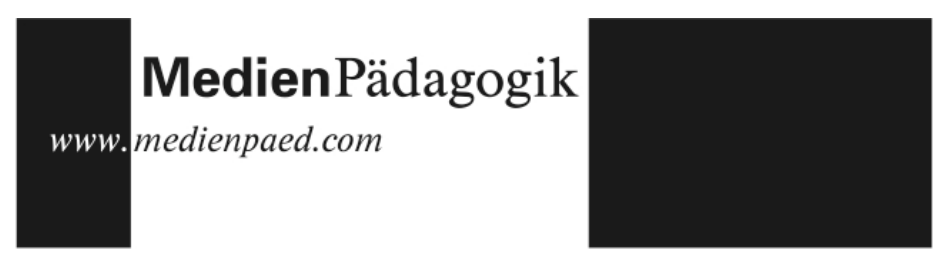

Rezensionen

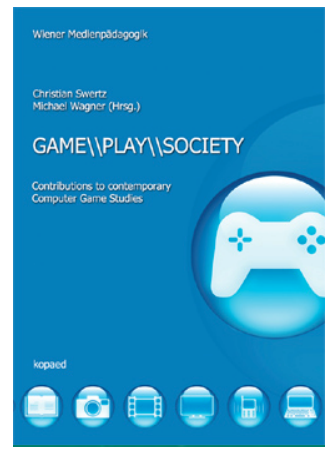

Christian Swertz, Michael Wagner

\title{
GAMEIIPLAYIISociety
}

Contributions to contemporary Computer Game Studies

München: kopaed, 2010. 294 Seiten

ISBN: 3-86736-240-8

$€ 19.80$

\section{Annäherungen an das Phänomen Computerspiel}

Spiele sind so alt wie die Menschheit selbst. Recht neu ist jedoch ist die akademische Annäherung. Das Entstehen der Game Studies liegt in der anhaltenden Entwicklung (technisch als auch gesellschaftlich) der Computerspiele begründet. Der im Rahmen der Konferenz «Future and Reality of Gaming» erschienene Sammelband GAMEIIPLAYIISOCIETY widmet sich diesem Thema und macht bereits im Titel deutlich, dass holistische Betrachtungen dem Thema angemessen sind. Daher ist die ausgeprägte Vielfalt der Annährungs- und Betrachtungsweisen eine logische Konsequenz.

Dieser Heterogenität geschuldet kann der Band nicht als direkte Fortführung eines spezifischen wissenschaftlichen Diskurses gesehen werden. Dennoch kristallisieren sich mit Blick auf die verwendete Literatur häufiger verwendete Autoren heraus. Erwähnenswert sind hier Jesper Juul, James Paul Gee und lan Bogost, die mit ihren Arbeiten maßgeblich zur Etablierung der Game Studies beigetragen haben. Ebenso wird an die Arbeiten von Jürgen Fritz und Christoph Klimmt angeknüpft, die vor allem die deutsche Beteiligung an der Videospielfoschung widerspiegeln.

Die Einzelbeiträge des Bandes sind dem Titel entsprechend in drei Kapitel unterteilt, die allerdings nicht als trennscharfe Kategorisierung verstanden werden dürfen, sondern eher als der Versuch, eine grobe Ordnung in die Bandbreite der Beiträge zu bringen. 


\section{Rezensionen}

Aus unterschiedlichen Perspektiven wird das MMORPG World of Warcraft betrachtet. Kramer analysiert die enormen sprachlichen Varietäten der Spieler innerhalb des Spiels in Bezug auf ihre zahlreichen Funktionen. Bliefenicht stellt sich der Frage, weshalb insbesondere World of Warcraft oft ein besonders hohes Abhängigkeitspotential unterstellt wird. Er nähert sich dem Spiel auf soziologischem Wege und argumentiert, dass eine Einordnung des Phänomens Computerspielsucht innerhalb gängiger Klassifikationssysteme nicht angemessen ist. World of Warcraft ist auch Untersuchungsgegenstand zweier Beiträge, die sich mit geschlechtsspezifischen Unterschieden in der Spielerschaft auseinandersetzen. Während Kolo und Müller quantitativ die unterschiedlichen Motivationskomponenten verschiedener Games untersuchen, stehen bei der qualitativen Analyse Hommedals die bevorzugten Genres der weiblichen Spieler im Vordergrund. Bauer, Fessler und Horvatic betrachten mit Die Stämme ein strukturähnliches Online-Game. Für sie stehen die Machtstrukturen des Spiels im Vordergrund, die durch das gemeinsame Spiel zwangsläufig entstehen, sollte es sich um progressive Spielergemeinschaften handeln. Die Ergebnisse sind dabei generell auf sämtliche Spiele übertragbar, in denen sich die Spieler zu Gilden oder Clans zusammenschließen.

Einige Autoren setzen sich mit pädagogischen Möglichkeiten von Computerspielen auseinander. Reng und Schoenau-Fog berichten von erfolgreichen Versuchen, technisches Wissen an Hochschulen durch den kreativen Schaffensprozess im Game-Design zu vermitteln. Einen Einblick in den formalen Charakter einer Umweltsimulationen mit den damit verbundenen pädagogischen Implikationen geben Vilaça, Torrão und Rodrigues. Lacasa und Martinez-Borda untersuchen, wie mit Hilfe des Klassikers Mario Bros schon bei Kindern die Fähigkeit zur Reflektion und Strategieentwicklung gefördert werden kann.

Darüber hinaus geben die Autoren des Bandes Einblick in weitere Forschungsfelder. Die Bandbreite der Beiträge reicht vom Phänomen des «Achievements» (Raczokwski, Schollar) über «Mediennostalgie» (Felzmann) bis hin zur «Videospiel-Distribution» in der post-kommunistischen Tschechei (Švelch). 
Dass es sich schwierig gestaltet, aus den Beträgen etwas Gemeinsames zu abstrahieren und eine gewisse Ordnung in diese zu bringen, ist der Tatsache geschuldet, dass die Game Studies in ihrem Etablierungsprozess noch eine unparadigmatische Leichtigkeit innewohnt, die es den Beteiligten erlaubt, sich explorativ und experimentell mit dem Gegenstand auseinanderzusetzen. Daher kann der Sammelband als eine Sondierung der Möglichkeiten verstanden werden, der keinerlei Anspruch auf Vollständigkeit erhebt. Wer diese sucht, wird eher bei den Werken der eingangsgenannten Autoren fündig.

Kritisch bleibt nach wie vor der scheinbare Unwille einiger deutscher Autoren, ihre Ergebnisse in englischer Sprache zu veröffentlichen. So wäre wünschenswert, dass die ausschließlich im Deutschen veröffentlichten Werke Jürgen Fritz' zumindest über die Sekundärliteratur der englisch- sprachigen globalen Forschergemeinde zugänglich gemacht werden.

Kelvin Autenrieth 